BMJ Open

Diabetes

Research

\& Care

\title{
Risk of cardiovascular event and mortality in relation to refill and guideline adherence to lipid-lowering medications among patients with type 2 diabetes mellitus in Sweden
}

\author{
Sofia Axia Karlsson, ${ }^{\oplus 1}$ Björn Eliasson, ${ }^{2}$ Stefan Franzén, ${ }^{3}$ Mervete Miftaraj, ${ }^{3}$ \\ Ann-Marie Svensson, ${ }^{3}$ Karolina Andersson Sundell ${ }^{1,4}$
}

To cite: Karlsson SA, Eliasson B, Franzén S, et al. Risk of cardiovascular event and mortality in relation to refill and guideline adherence to lipid-lowering medications among patients with type 2 diabetes mellitus in Sweden. BMJ Open Diab Res Care 2019;7:e000639. doi:10.1136/ bmjdrc-2018-000639

- Additional material is published online only. To view please visit the journal online (http://dx.doi.org/10.1136/ bmjdrc-2018-000639).

Received 5 December 2018 Revised 15 February 2019 Accepted 4 March 2019
Check for updates

(C) Author(s) (or their employer(s)) 2019. Re-use permitted under CC BY. Published by BMJ.

For numbered affiliations see end of article.

Correspondence to Sofia Axia Karlsson; sofia.karlsson@gu.se

\section{ABSTRACT}

Objective To analyze the risk of cardiovascular (CV) events and mortality in relation to adherence to lipid-lowering medications by healthcare centers and patients with type 2 diabetes mellitus (T2DM).

Research design and methods We included 121914 patients (12\% secondary prevention) with T2DM reported by 1363 healthcare centers. Patients initiated lipidlowering medications between July 2006 and December 2012 and were followed from cessation of the first filled supply until multidose dispensed medications, migration, CV events, death or December 2016. The study period was divided into 4-month intervals through 2014 , followed by annual intervals through 2016. Adherence measures were assessed for each interval. Patients' (refill) adherence was measured using the medication possession ratio (MPR). Healthcare centers' (guideline) adherence represented the prescription prevalence of lipid-lowering medications according to guidelines. The risk of CV events and mortality was analyzed for each interval using Cox proportional hazard regression and Kaplan-Meier.

Results Compared with high-adherent patients (MPR $>80 \%$ ), low-adherent primary prevention patients (MPR $\leq 80 \%$ ) showed higher risk of all outcomes: $44 \%-51 \%$ for CV events, doubled for all-cause mortality and $79 \%-90 \%$ for CV mortality. Corresponding risks for low-adherent secondary prevention patients were $17 \%-19 \%$ for CV events, $88 \%-97 \%$ for all-cause and $66 \%-79 \%$ for CV mortality. Primary prevention patients treated by lowadherent healthcare centers (guideline adherence $<48 \%$ ) had a higher risk of CV events and CV mortality. Otherwise, no difference in the risk of CV events or mortality was observed by guideline adherence level.

Conclusions Our results demonstrate the importance of high refill adherence and thus the value of individualized care among patients with T2DM.

\section{INTRODUCTION}

Type 2 diabetes mellitus (T2DM) is a multifactorial disease that requires intensive glycemic control and treatment for comorbidities and complications to reduce the increased risk of cardiovascular disease (CVD) and mortality. ${ }^{1-3}$

\section{Significance of this study}

What is already known about this subject?

- High refill adherence to lipid-lowering medications associates with lower risk of cardiovascular event and mortality among patients with type 2 diabetes mellitus; the impact of the treating healthcare centers' adherence to lipid-lowering guidelines is uncertain.

\section{What are the new findings?}

- We found patients' refill adherence to lipid-lowering medications to have greater impact than adherence to guidelines by the treating healthcare center in terms of prevention of cardiovascular event and mortality in both primary and secondary prevention patients.

How might these results change the focus of research or clinical practice?

- Our results emphasize the value of individualized care and the importance of maximizing refill adherence to lipid-lowering medications among patients with type 2 diabetes mellitus.

Despite declining mortality rates among Swedish patients with T2DM, the risk is still higher than among patients without diabetes mellitus, and CVD remains the major cause of death. ${ }^{4}$ Improved control of low-density lipoprotein (LDL) cholesterol with lipid-lowering medications has been shown to reduce the risk of CVD and mortality among patients with T2DM. ${ }^{5-8}$ Thus, national ${ }^{9}$ and international ${ }^{110}$ treatment guidelines recommended lipid-lowering medications to patients with T2DM to lower LDL cholesterol below $2.5 \mathrm{mmol} / \mathrm{L}$. For patients with established CVD, LDL cholesterol below $1.8 \mathrm{mmol} / \mathrm{L}$ is desirable. 
Adherence is defined as the extent to which individuals follow agreed recommendations. ${ }^{11}$ Previous studies have shown that patients with T2DM with adherence of at least $80 \%$ to lipid-lowering medications face a lower risk of CVD and mortality than those with adherence below 80\%. ${ }^{5681213}$ Among multiple levels of adherence to lipid-lowering medications, a gradual increase in CVD risk was observed with declining adherence by patients with T2DM. ${ }^{6}$

Previous studies have reported adherence by healthcare providers to recommended treatment guidelines at $24 \%-80 \%$ among patients with diabetes mellitus. ${ }^{14-19}$ Patients with T2DM or established CVD were more likely to receive lipid-lowering medications. However, little is known about the impact of healthcare provider adherence to lipid-lowering medications on the risk of cardiovascular (CV) events or mortality among patients with T2DM.

Our primary objective was to analyze the risk of CV events and mortality in relation to T2DM patients' adherence to lipid-lowering medications and providers' adherence to lipid-lowering guidelines. Our secondary objective was to identify differences in the risk of CV events and mortality among various patient characteristics.

\section{METHODS}

Data sources

The unique Swedish personal identity number was used to link individual-based data from six national registers. Filled prescriptions were collected from the Swedish Prescribed Drug Register (SPDR), which contains information about all prescriptions filled at Swedish pharmacies since July $2005 .{ }^{20}$ Swedish prescriptions include information about the medication, patient, prescriber and the prescribed daily dosage. Clinical and health-related data about risk factors and complications of diabetes, as well as healthcare center characteristics, were collected from the Swedish National Diabetes Register (NDR), which contains patient information reported by physicians and nurses at hospitals and primary care clinics nationwide. ${ }^{21}$ Healthcare providers were identified at the center level, not by individual practitioners. Data concerning CV events were collected from the Swedish National Patient Register, which contains information about inpatient and outpatient care in Sweden. ${ }^{22}$ The date and cause of death were collected from the Cause of Death Register. ${ }^{23}$ Information about primary tumors was collected from the Swedish Cancer Registry. ${ }^{24}$ Individual data about socioeconomic status were collected from the Longitudinal Integration Database for Health Insurance and Labour Market Studies (LISA). ${ }^{25}$

\section{Study population and period}

Patients age 18 years or older with a clinical T2DM diagnosis ${ }^{26}$ who had filled at least one prescription for lipid-lowering medications between July 1, 2006 and December 31, 2012 were eligible for inclusion (figure 1).

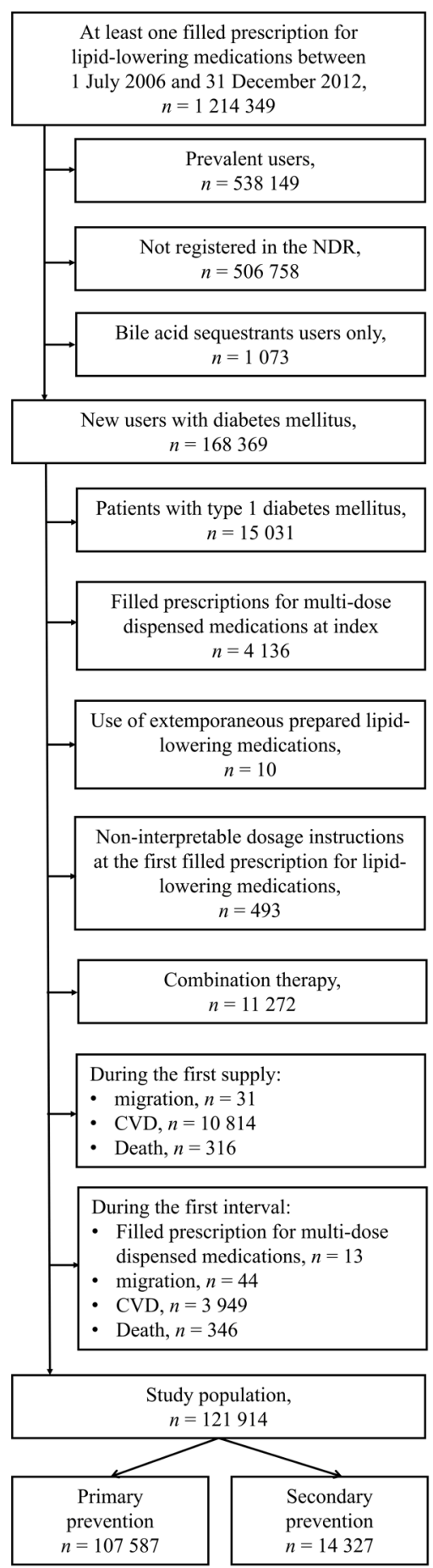

Figure 1 Inclusion and exclusion of the study population. CVD, cardiovascular disease; NDR, National Diabetes Register.

Prescriptions for bile acid sequestrants were excluded since the indication is typically not hyperlipidemia. ${ }^{27}$ The date of the first filled prescription was defined as the index date. To establish a new-user design, patients who had filled prescriptions for lipid-lowering medications within 1 year prior to the index date were regarded as prevalent users and excluded. Patients who filled prescriptions for a lipid-lowering combination therapy or lipid-lowering 
extemporaneous preparation that lacked information about package size were also excluded. Combination therapy has been described elsewhere. ${ }^{6}{ }^{28}$ Patients who experienced CVD prior to or on the index date were classified as prescribed lipid-lowering secondary prevention; all other patients were defined as primary prevention.

Patients were followed from cessation of the first filled supply (baseline date) until the first filled prescription for multi-dose dispensed medications, migration, CV event, death or December 31, 2016. The study period was broken down into subsequent intervals of 122 days until December 31, 2014, followed by annual intervals until December 31, 2016.

The healthcare center at baseline was assigned by selecting the entry in the NDR closest to the baseline date and remained the same throughout the study period unless the NDR indicated otherwise. A comparison between the assigned healthcare center from the NDR with the healthcare provider of the first filled lipid-lowering prescription in the SPDR showed agreement of $98 \%$ for county council and $87 \%$ for type of care.

\section{Refill adherence}

Patients' (refill) adherence to lipid-lowering medications was assessed with data from the SPDR. We assumed that patients initiated medication use on the index date. We measured refill adherence with the medication possession ratio (MPR), representing the proportion of days with medications available. The supply of each prescription was calculated by dividing the number of tablets filled by the daily dosage stated as a free-text variable by the prescriber. Interpretation of the free-text variable to obtain the daily dosage has been described elsewhere. ${ }^{628}$ Overlapping supplies for the same substance and strength were added to the latter supply. Overlapping supplies for different substances or strengths were deleted. MPR was assessed for each subsequent interval and categorized as high or low based on the common cut-off of $80 \% .{ }^{29} 30$

\section{Guideline adherence}

Based on data from the NDR, we assessed healthcare center adherence to national lipid-lowering prescription guidelines. Guideline adherence was defined as the prescription prevalence of lipid-lowering medications among patients with T2DM and LDL cholesterol above the recommended target levels existing at the time for the study $\left(\geq 2.5 \mathrm{mmol} / \mathrm{L}\right.$ for primary prevention ${ }^{31}$ and $\geq 1.8 \mathrm{mmol} / \mathrm{L}$ for secondary prevention ${ }^{1}{ }^{10}$ ). Between 2007 and 2014, guideline adherence was assessed for each healthcare center and year, for primary and secondary prevention patients separately.

Guideline adherence was linked to patient intervals based on the year in which the interval started. For intervals starting in 2006, guideline adherence for 2007 was used. For healthcare centers that lacked information about adherence, we imputed the preceding year's figure or the mean annual adherence for the county council and type of care. Guideline adherence was categorized as high or low based on a cut-off that represented the median for primary $(48 \%)$ and secondary prevention $(78 \%)$.

\section{Outcomes}

The outcomes of interest were CV events and mortality. A CV event was defined as a diagnosis of unstable angina pectoris, myocardial infarction (including percutaneous coronary intervention, coronary artery bypass grafting), stroke or ischemic heart disease. All-cause mortality was defined as death from any cause. CV mortality was defined as death from a cause of CVD or a CV event entered in the National Patient Register within 28 days prior to death. Starting from the second interval, the risk of CV events and mortality was analyzed for each interval until migration, CV event, death or December 31, 2016.

\section{Covariates}

Covariates regarded as potential confounders included sex, age, socioeconomic status (country of birth, marital status, education level, employment status, profession, income), concurrent medications (filled prescriptions for diabetes medications, anticoagulants and antihypertensives), and clinical and health-related characteristics (diabetes duration, hemoglobin A1c [HbAlc], estimated glomerular filtration rate [eGFR], blood pressure, cholesterol values, microalbuminuria, macroalbuminuria, kidney disease, cancer, body mass index [BMI], physical activity and smoking). These covariates have previously been shown to be important factors when analyzing adherence, as well as the risk of CV events and mortality. 628

Sex, age and socioeconomic characteristics were collected from the LISA database. Sex and country of birth were regarded as constant, and age was based on the year of birth. Information about marital status, education level, employment status, profession and income was collected prior to or during the baseline year. Income was regarded as a continuous variable. The remaining socioeconomic variables included the following categories: country of birth: Sweden, other Nordic country, other European Union (EU)-15 country or the Soviet Union, rest of Europe, the Americas, Asia or Oceania, or unknown; marital status: unmarried, married or registered partner, divorced, or widow/widower; education level: compulsory school or lower, upper secondary school, or postsecondary; employment status: unemployed, employed or retired; profession: upper whitecollar, lower white-collar, blue-collar, or other.

At baseline, cancer and kidney disease were defined as diagnosis within 5 years prior to the baseline date and were collected from the Swedish Cancer Registry and the National Patient Register, respectively. Cancer diagnosis included primary tumors, while kidney disease included acute or chronic kidney failure, as well as glomerular or renal complication due to T2DM.

Filled prescriptions for diabetes medications (anatomical therapeutic chemical (ATC): A10), anticoagulants 
(ATC: B01), and antihypertensives (ATC: C02, C03, C04, $\mathrm{C} 05, \mathrm{C} 07, \mathrm{C} 08$, and C09) were collected from the SPDR. Filled prescriptions within 12 months prior to the baseline date were considered concurrent use.

The remaining clinical and health-related characteristics were collected from the NDR. At baseline, data were collected between 24 months prior to and 14 days after the baseline date by selecting the value closest to baseline. Diabetes duration was based on the year of birth and diabetes diagnosis. Microalbuminuria and macroalbuminuria were dichotomously categorized. BMI and eGFR were categorized according to recommended references values. ${ }^{32}{ }^{33} \mathrm{HbA1c}$, blood pressure and cholesterol levels were categorized as high or low according to recommended target values at the time of the study. ${ }^{31}$ Physical activity was defined as a 30 min walk or equivalent, categorized as less than once a week, 1-2 times a week, 3-5 times a week or daily. Smoking was dichotomized and defined as at least one cigarette / pipe daily or having quit within the past 3 months.

A total of $23 \%$ of patient characteristics at baseline were missing: $4 \%$ of socioeconomic status and $43 \%$ of clinical and health-related characteristics. No information was missing about age, sex or concurrent medications. Missing information at baseline was replaced with multiple imputations. Potential confounders (except for cancer) were assessed for each interval during the study period by assuming the imputed baseline value until the information had been updated in the registers.

\section{Sensitivity analyses}

To evaluate the cut-offs used to categorize refill and guideline adherence as high or low, new ones were set. For refill adherence, cut-offs of $60 \%, 70 \%$, and $90 \%$ were applied to evaluate the $80 \%$ level that had been used to define patients as low-adherent or high-adherent. For guideline adherence, new cut-offs were set at $30 \%$ and $60 \%$ (from $48 \%$ ) for primary prevention and $50 \%$ and $90 \%$ (from $78 \%$ ) for secondary prevention. The results of the statistical analyses were compared between the cut-offs.

To estimate the impact of multiple imputations, the risk of CVD and mortality was assessed and compared between complete cases and imputed data.

\section{Statistical analyses}

The association between refill (MPR) and guideline adherence was analyzed by means of general linear regression. Multivariate imputations by chained equations (MICE) were used to replace missing information among baseline variables; 10 imputed data sets with 10 iterations each were generated. The risk of CV events and mortality was analyzed for each interval based on the 10 imputed data sets with Cox proportional hazard regression and Kaplan-Meier, adjusted for potential confounders. Covariates and guideline adherence for one interval were regarded as potential confounders for the subsequent interval of MPR measures. Similarly, MPR for one interval was regarded as the exposure for the subsequent interval of outcome measures (online supplementary figure S1). HRs generated for each imputed data set were pooled and one final set was established. The same procedure was performed to assess adjusted and pooled survival estimates and obtain Kaplan-Meier survival curves for CV events and mortality. All hypothesis tests were evaluated at a 5\% significance level.

To present baseline characteristics for the study population based on imputed data, the mean values of continuous variables and the most frequent category of categorical variables were obtained from the imputed data sets. These generated baseline values were used to descriptively present the study population but were not used in the statistical analyses.

Multiple imputations were performed in R V.3.3.2. using the MICE package. ${ }^{35}$ All other data management and statistical analyses were performed with SAS V.9.4 software.

\section{RESULTS}

\section{Study population and period}

A total of 121914 patients with T2DM were included (figure 1). Of them, a total of $11.8 \%$ started on lipid-lowering secondary prevention. The mean age was 63 years, $57 \%$ were men and the mean diabetes duration was 5 years (table 1). Approximately $80 \%$ were born in Sweden and more than 50\% were married. Among primary prevention patients, the mean age was 62 years, 56\% were men and the mean diabetes duration was 5 years. Among secondary prevention patients, the mean age was 70 years, $61 \%$ were men and the mean diabetes duration was 6.5 years.

A total of $67 \%$ of primary prevention patients filled prescriptions for diabetes medications, 33\% for anticoagulants and $72 \%$ for antihypertensives. A total of $61 \%$ of secondary prevention patients filled prescriptions for diabetes medications, $89 \%$ for anticoagulants and $92 \%$ for antihypertensives. Cancer and kidney disease prior to the index date had been diagnosed in $4 \%$ and $1 \%$ of primary prevention patients, respectively, and $5 \%$ and $3 \%$ of secondary prevention patients, respectively. A total of $17 \%$ of primary prevention patients were physically active less than once a week and $12 \%$ were smokers. The corresponding figures for secondary prevention patients were $22 \%$ and $8 \%$. For both prevention groups, the mean HbAlc was above $52 \mathrm{mmol} / \mathrm{mol}$, the mean BMI was approximately 30 and the mean LDL cholesterol was above $3 \mathrm{mmol} / \mathrm{L}$.

The mean supply for the first filled prescription was $87( \pm 28)$ days for primary prevention patients and 90 $( \pm 25)$ days for secondary prevention patients. Among primary prevention patients, the mean study period was $2389( \pm 855)$ days for CVD and $2545( \pm 761)$ for mortality. Among secondary prevention patients, the mean study period was $1690( \pm 1071)$ days for CVD and $2416( \pm 885)$ days for mortality. A total of $79 \%(n=84562)$ of primary 
Table 1 Baseline characteristics (imputed data) of all 121914 new users of lipid-lowering medications with type 2 diabetes mellitus and by prevention type

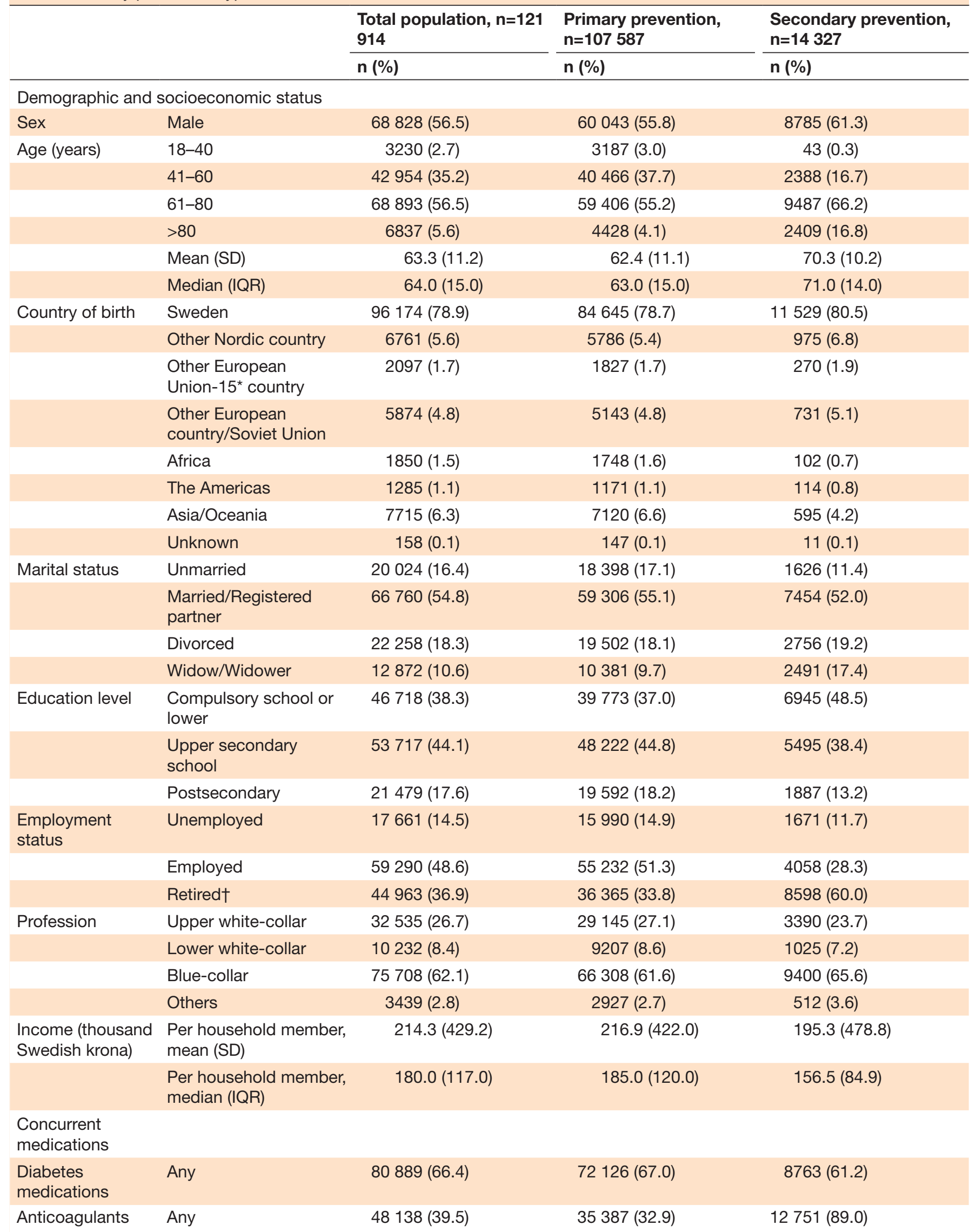


Table 1 Continued

\begin{tabular}{|c|c|c|c|c|}
\hline & & $\begin{array}{l}\text { Total population, } n=121 \\
914\end{array}$ & $\begin{array}{l}\text { Primary prevention, } \\
\mathrm{n}=107587\end{array}$ & $\begin{array}{l}\text { Secondary prevention, } \\
n=14327\end{array}$ \\
\hline & & n (\%) & n (\%) & n (\%) \\
\hline Clinical and health- & -related characteristics & & & \\
\hline Diabetes duration & Mean (SD) & $5.0(6.4)$ & $4.8(6.1)$ & $6.5(7.7)$ \\
\hline $\mathrm{HbA} 1 \mathrm{c}, \mathrm{mmol} / \mathrm{mol}$ & $<42(<5)$ & $9706(8.0)$ & $8754(8.1)$ & $952(6.6)$ \\
\hline & $42-52(5-6)$ & $61162(50.2)$ & 53525 (49.8) & 7637 (53.3) \\
\hline & $>52(>6)$ & $51046(41.9)$ & $45308(42.1)$ & $5738(40.1)$ \\
\hline$(\mathrm{mL} / \mathrm{min} / 1.73 \mathrm{~m} \dagger)$ & $\geq 60$ & 112308 (92.1) & 100066 (93.0) & 12242 (85.5) \\
\hline & Mean (SD) & $85.3(20.3)$ & $86.3(20.2)$ & 77.5 (18.9) \\
\hline & Median (IQR) & $84.3(22.6)$ & $85.3(22.4)$ & $77.4(21.1)$ \\
\hline BMI & $<18.5$ & $173(0.1)$ & $155(0.1)$ & $18(0.1)$ \\
\hline$(\mathrm{kg} / \mathrm{m}+)$ & $18.5-24.9$ & $10015(8.2)$ & $8795(8.2)$ & $1220(8.5)$ \\
\hline & $25.0-29.9$ & 52074 (42.7) & 45169 (42.0) & 6905 (48.2) \\
\hline & $\geq 30.0$ & 59652 (48.9) & 53468 (49.7) & $6184(43.2)$ \\
\hline & Mean (SD) & $30.3(4.5)$ & $30.4(4.5)$ & $29.8(4.2)$ \\
\hline & Median (IQR) & $29.9(4.8)$ & $30.0(4.9)$ & $29.5(4.2)$ \\
\hline & $\geq 80$ & 63776 (52.3) & 57826 (53.8) & $5950(41.5)$ \\
\hline & Mean (SD) & $79.1(8.0)$ & $79.3(8.0)$ & $77.4(7.8)$ \\
\hline & Median (IQR) & $80.0(8.0)$ & $80.0(8.5)$ & $78.2(7.2)$ \\
\hline LDL cholesterol & $<2.5$ & $11556(9.5)$ & 9937 (9.2) & 1619 (11.3) \\
\hline & $\geq 2.5$ & 110358 (90.5) & 97650 (90.8) & 12708 (88.7) \\
\hline & Mean (SD) & $3.4(0.7)$ & $3.4(0.7)$ & $3.2(0.7)$ \\
\hline & Median (IQR) & $3.4(0.7)$ & $3.4(0.7)$ & $3.3(0.7)$ \\
\hline $\begin{array}{l}\text { HDL cholesterol } \\
(\mathrm{mmol} / \mathrm{L})\end{array}$ & $\begin{array}{l}<1.0 \text { (men) } /<1.3 \\
\text { (women) }\end{array}$ & 30489 (25.0) & 27425 (25.5) & 3064 (21.4) \\
\hline & $\begin{array}{l}\geq 1.0 \text { (men)/ } \geq 1.3 \\
\text { (women) }\end{array}$ & 91425 (75.0) & $80162(74.5)$ & $11263(78.6)$ \\
\hline & Mean (SD), men/women & $1.2(0.3) / 1.4(0.3)$ & $1.2(0.3) / 1.4(0.2)$ & $1.2(0.2) / 1.4(0.3)$ \\
\hline & $\begin{array}{l}\text { Median (IQR), men/ } \\
\text { women }\end{array}$ & $1.2(0.3) / 1.3(0.3)$ & $1.1(0.3) / 1.4(0.3)$ & $1.2(0.3) / 1.4(0.3)$ \\
\hline $\begin{array}{l}\text { Triglycerides } \\
(\mathrm{mmol} / \mathrm{L})\end{array}$ & $<2.0$ & 73909 (60.6) & 64400 (59.9) & 9509 (66.4) \\
\hline
\end{tabular}




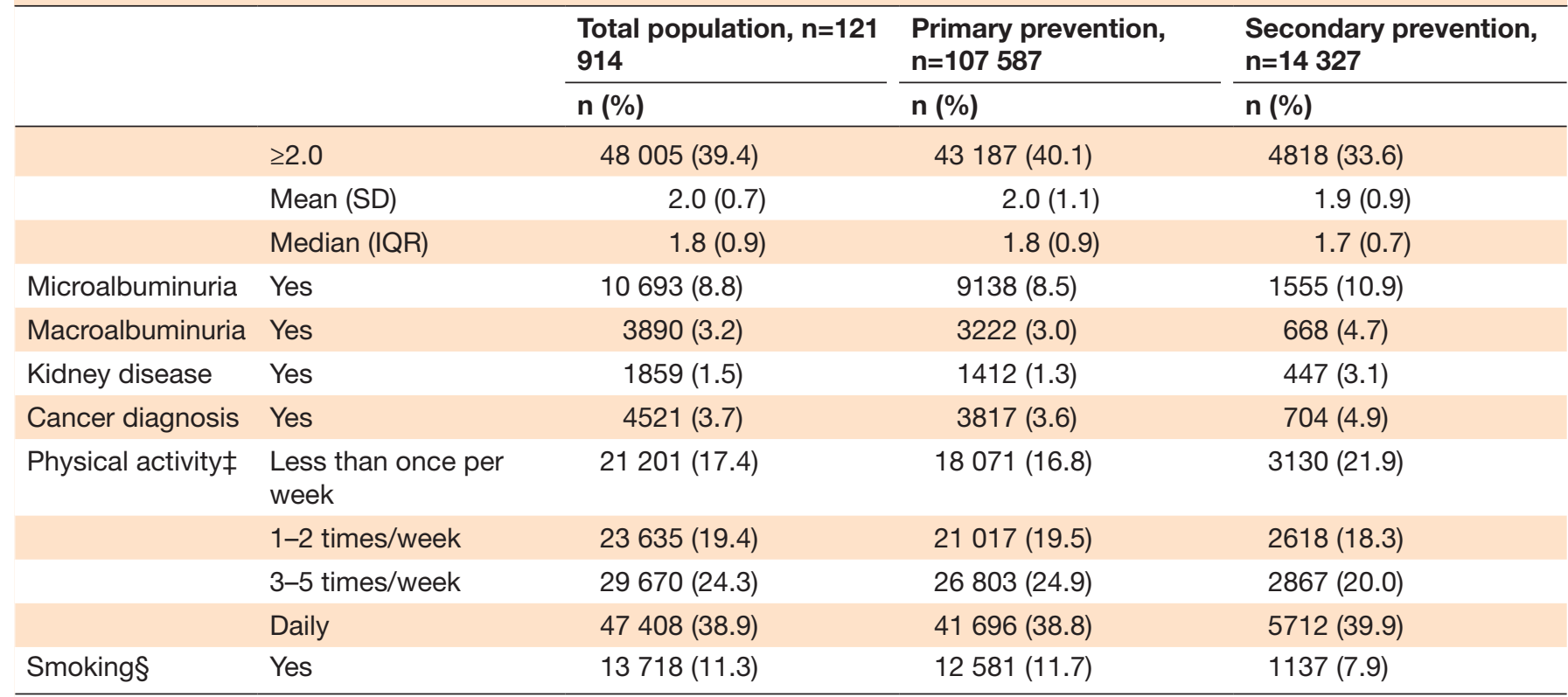

*Includes Belgium, Denmark, Germany, Greece, Spain, France, Ireland, Italy, Luxembourg, The Netherlands, Austria, Portugal, Finland, Sweden and Great Britain.

†If age 65 years or older and unemployed.

$\ddagger 30$ min walk or equivalent.

$\S A$ teast one cigarette or pipe daily or quit smoking within 3 months.

BMI, body mass index; HDL, high-density lipoprotein; HbA1c, hemoglobin A1c; LDL, low-density lipoprotein; eGFR, estimated glomerular filtration rate.

prevention patients were not censored or experienced any outcome during the study period and were thus followed until December 31, 2016. The corresponding figure for secondary prevention patients was $38 \%(n=5413)$.

For CVD measures a total of $1 \%(n=1095)$ of primary prevention patients and $8 \%(n=1115)$ of secondary prevention patients were followed through one interval of outcome. For mortality measures, less than $1 \%$ of all patients were followed through one interval only.

\section{Refill and guideline adherence}

Among patients with primary prevention, the overall mean refill adherence (MPR) was $63.6 \%$, ranging from $60.7 \%$ to $70.0 \%$ throughout the study period. The mean refill adherence (MPR) for secondary prevention patients ranged from $55.6 \%$ to $73.1 \%$ throughout the study period, averaging $61.2 \%$. A total of $6 \%$ of primary prevention patients and $10 \%$ of secondary prevention patients filled only their initial prescription.

A total of 1363 healthcare centers, $93 \%$ of which were primary care, were included. The overall mean annual guideline adherence was $49 \%$, ranging from $48 \%$ to $49 \%$ throughout the study period for primary prevention patients, and $76 \%$, ranging from $73 \%$ to $77 \%$ throughout the study period for secondary prevention patients.

With each percentage of guideline adherence, refill adherence increased by $0.01 \%$ for primary prevention patients and $0.002 \%$ for secondary prevention patients $(\mathrm{p}<0.0001)$.

\section{CV events and mortality}

During the study period, 21396 patients experienced CV events and 17282 died. Almost 33\% of all deaths were caused by CVD. A total of $13 \%$ of primary prevention patients experienced $\mathrm{CV}$ events and $12 \%$ died during the study period. A total of $51 \%$ of secondary prevention patients experienced CV events and 32\% died during the study period. Death due to CVD accounted for $28 \%$ and $47 \%$ of all deaths among primary and secondary prevention patients, respectively.

Adjusted for potential confounders, primary prevention patients with low refill adherence had a $44 \%-51 \%$ higher risk of CV events, and 79\%-92\% higher risk of CV mortality and doubled risk of all-cause mortality (table 2). Primary prevention patients with low refill adherence who were being treated by healthcare centers with low guideline adherence faced an approximately $9 \%$ higher risk of $\mathrm{CV}$ events. Primary prevention patients who were being treated by healthcare centers with low guideline adherence had a 10\%-18\% higher risk of CV mortality.

Secondary prevention patients with low refill adherence had a 17\%-19\% higher risk of CV events, 88\%-97\% higher risk of all-cause mortality and $66 \%-79 \%$ higher risk of $\mathrm{CV}$ mortality than those with high refill adherence (table 2). There was no statistically significant difference in the risk of $\mathrm{CV}$ events and mortality between guideline adherence levels among secondary prevention patients.

In general, the risk of CV events and mortality was higher with increasing age, diabetes duration and 


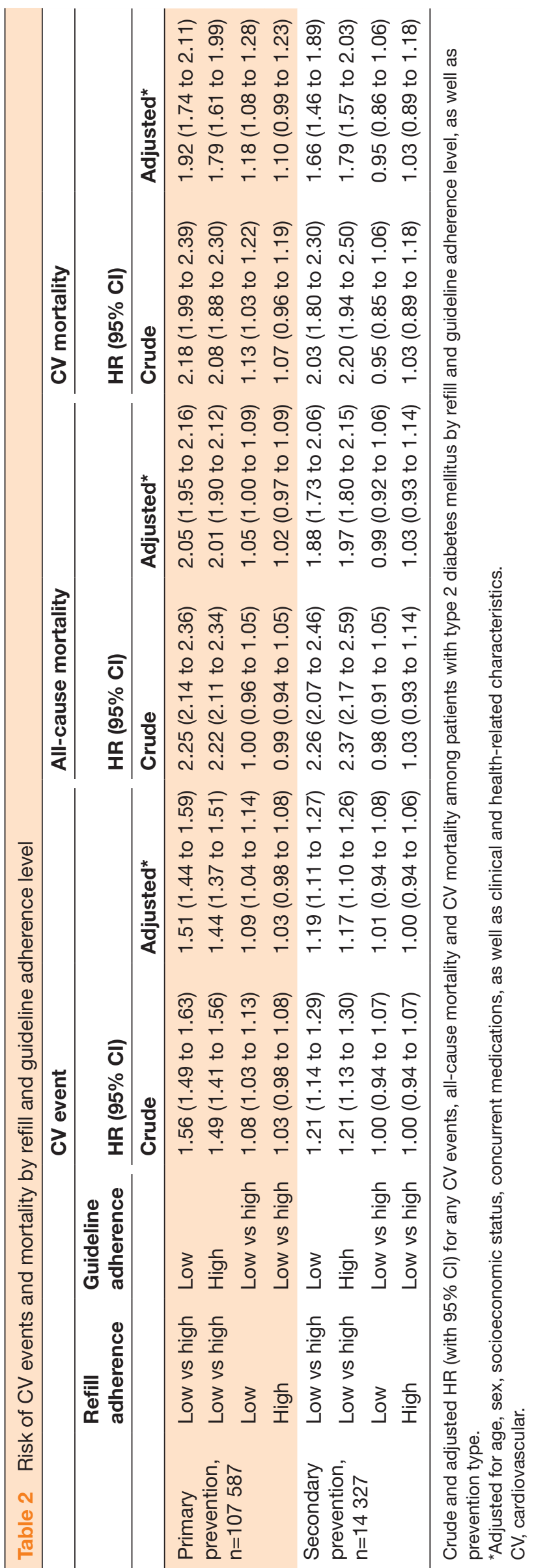

HbA1c, as well as among patients with low kidney function (kidney disease, microalbuminuria or macroalbuminuria), physical activity less than twice a week, smokers and men (table 3). A lower risk was observed among patients who had not filled prescriptions for anticoagulants or antihypertensives, as well as among patients born outside of Sweden.

Primary prevention patients who had not filled prescriptions for antihypertensives had a lower risk of mortality unlike those who had not filled a prescription for diabetes medications that associated with increased risk. Furthermore, the risk of CV events and mortality was associated with lower income and unemployment. Among secondary prevention patients, increasing high-density lipoprotein cholesterol was associated with a lower risk of all three outcomes.

Compared with low-adherent patients, the KaplanMeier survival curves showed higher survival of CV events and mortality among high-adherent patients (figure 2).

Primary prevention patients who were being treated at healthcare centers with low guideline adherence had lower survival of CV events than those who were being treated at high-adherent centers. Among high-adherent primary prevention patients, there was a tendency toward higher survival of $\mathrm{CV}$ events if they were being treated at high-adherent centers than at low-adherent centers. Among secondary prevention patients, guideline adherence had little or no impact on survival of CV events or mortality.

\section{Sensitivity analyses}

When the refill adherence cut-off alternated between $60 \%, 80 \%, 70 \%$, and $90 \%$, or when the guideline adherence cut-off was set to $30 \%$ or $60 \%$ for primary prevention and $50 \%$ or $90 \%$ for secondary prevention, the risk of CV events and mortality showed a consistent pattern, that is, low-adherent patients faced a higher risk than high-adherent patients, independent of guideline adherence.

Adjusted for potential confounders, the risk of $\mathrm{CV}$ events and mortality was similar between complete cases and imputed data: low-adherent patients had a higher risk of CV events and mortality than high-adherent patients, independent of guideline adherence. Furthermore, low-adherent primary prevention patients who were being treated at low-adherent healthcare centers had a greater risk of an outcome than those treated at high-adherent healthcare centers. Additionally, in complete case analysis, low-adherent secondary prevention patients treated by low-adherent healthcare centers had lower risk of CV mortality compared with those treated by high-adherent healthcare centers.

\section{DISCUSSION}

In this nationwide study of more than 120000 patients with T2DM, we analyzed the risk of CV events and mortality in relation to patient refill adherence to lipid-lowering medications and healthcare center 
Table 3 Risk of CV events and mortality for patient characteristics

\begin{tabular}{|c|c|c|c|c|c|c|}
\hline & \multicolumn{3}{|c|}{ Primary prevention, n=107 587} & \multicolumn{3}{|c|}{ Secondary prevention, $n=14327$} \\
\hline & CV events & All-cause mortality & CV mortality & CV events & All-cause mortality & CV mortality \\
\hline & HR (95\% Cl) & HR (95\% Cl) & HR (95\% Cl) & HR $(95 \% \mathrm{Cl})$ & HR $(95 \% \mathrm{Cl})$ & HR $(95 \% \mathrm{Cl})$ \\
\hline \multicolumn{7}{|l|}{ Sex, ref=female } \\
\hline Male & 1.54 (1.48 to 1.60$)$ & $1.43(1.37$ to 1.49$)$ & $1.62(1.50$ to 1.75$)$ & 1.35 (1.28 to 1.42$)$ & 1.48 (1.38 to 1.59$)$ & $1.62(1.46$ to 1.80$)$ \\
\hline \multicolumn{7}{|c|}{ Age (years), ref $=61-80$} \\
\hline $18-60$ & 0.61 (0.58 to 0.65$)$ & $0.45(0.42$ to 0.49$)$ & 0.45 (0.38 to 0.52$)$ & 0.95 (0.87 to 1.05$)$ & $0.50(0.41$ to 0.61$)$ & $0.48(0.36$ to 0.65$)$ \\
\hline$>80$ & 1.46 (1.39 to 1.54$)$ & 2.19 (2.09 to 2.29$)$ & 2.32 (2.13 to 2.52$)$ & 1.35 (1.27 to 1.43$)$ & 2.13 (1.99 to 2.29$)$ & 2.35 (2.12 to 2.61$)$ \\
\hline \multicolumn{7}{|c|}{ Country of birth, ref=Sweden } \\
\hline $\begin{array}{l}\text { Other Nordic } \\
\text { country }\end{array}$ & 1.18 (1.11 to 1.27$)$ & $1.02(0.95$ to 1.10$)$ & $1.10(0.96$ to 1.25$)$ & 1.14 (1.04 to 1.25$)$ & 0.99 (0.88 to 1.12$)$ & 1.06 (0.89 to 1.26$)$ \\
\hline $\begin{array}{l}\text { Other European } \\
\text { Union- } 15^{\star} \text { country }\end{array}$ & 0.89 (0.78 to 1.01$)$ & $0.82(0.72$ to 0.94$)$ & 0.77 (0.59 to 1.01$)$ & 1.18 (1.00 to 1.39$)$ & 1.07 (0.87 to 1.32$)$ & $1.22(0.91$ to 1.63$)$ \\
\hline $\begin{array}{l}\text { Other European } \\
\text { country/Soviet } \\
\text { Union }\end{array}$ & $1.11(1.03$ to 1.20$)$ & $0.89(0.81$ to 0.97$)$ & $0.86(0.72$ to 1.03$)$ & $1.08(0.97$ to 1.21$)$ & $0.86(0.74$ to 1.00$)$ & $0.84(0.67$ to 1.05$)$ \\
\hline Africa & 0.68 (0.57 to 0.81$)$ & $0.47(0.37$ to 0.61$)$ & 0.41 (0.24 to 0.69$)$ & 0.97 (0.72 to 1.30$)$ & $0.92(0.59$ to 1.43$)$ & $1.02(0.54$ to 1.90$)$ \\
\hline The Americas & 0.84 (0.69 to 1.01$)$ & 0.64 (0.51 to 0.81$)$ & 0.64 (0.41 to 1.00$)$ & 0.70 (0.52 to 0.96$)$ & 0.88 (0.60 to 1.30$)$ & 0.73 (0.39 to 1.37$)$ \\
\hline Asia/Oceania & 1.15 (1.07 to 1.25$)$ & 0.48 (0.43 to 0.54$)$ & $0.63(0.51$ to 0.77$)$ & 1.15 (1.02 to 1.30$)$ & 0.71 (0.58 to 0.87$)$ & 0.74 (0.55 to 1.00$)$ \\
\hline Unknown & $1.38(0.91$ to 2.11$)$ & $0.82(0.44$ to 1.54$)$ & 0.89 (0.29 to 2.79$)$ & $1.47(0.70$ to 3.10$)$ & NA & NA \\
\hline \multicolumn{7}{|c|}{ Marital status, ref=married } \\
\hline Unmarried & 0.87 (0.83 to 0.92$)$ & $1.18(1.12$ to 1.25$)$ & 1.41 (1.27 to 1.57$)$ & 0.89 (0.82 to 0.97$)$ & 1.26 (1.13 to 1.40$)$ & $1.46(1.26$ to 1.69$)$ \\
\hline Divorced & 1.04 (0.99 to 1.09$)$ & $1.11(1.05$ to 1.17$)$ & 1.17 (1.06 to 1.30$)$ & 1.01 (0.95 to 1.08$)$ & 1.05 (0.96 to 1.14$)$ & $1.06(0.93$ to 1.21$)$ \\
\hline Widow(er) & 1.14 (1.09 to 1.20$)$ & $1.20(1.14$ to 1.26$)$ & $1.38(1.25$ to 1.51$)$ & 1.06 (0.99 to 1.13$)$ & 1.20 (1.11 to 1.29$)$ & 1.19 (1.06 to 1.33$)$ \\
\hline \multicolumn{7}{|c|}{ Education level, ref=mandatory school } \\
\hline $\begin{array}{l}\text { Upper secondary } \\
\text { school }\end{array}$ & 0.95 (0.91 to 0.98$)$ & 0.93 (0.89 to 0.96$)$ & 0.91 (0.84 to 0.98$)$ & 0.98 (0.93 to 1.03$)$ & 0.91 (0.85 to 0.97$)$ & $0.89(0.81$ to 0.98$)$ \\
\hline $\begin{array}{l}\text { Postsecondary } \\
\text { education }\end{array}$ & $0.92(0.87$ to 0.98$)$ & $0.86(0.80$ to 0.92$)$ & 0.81 (0.71 to 0.92$)$ & $0.98(0.90$ to 1.07$)$ & $0.93(0.83$ to 1.04$)$ & $0.86(0.73$ to 1.02$)$ \\
\hline \multicolumn{7}{|c|}{ Employment status, ref=employed } \\
\hline Unemployed & 1.15 (1.08 to 1.23$)$ & 1.46 (1.34 to 1.59$)$ & $1.42(1.21$ to 1.67$)$ & $1.05(0.95$ to 1.17$)$ & 1.26 (1.06 to 1.51$)$ & $1.12(0.86$ to 1.45$)$ \\
\hline Retired $\dagger$ & $1.30(1.24$ to 1.37$)$ & 1.93 (1.82 to 2.04$)$ & $1.93(1.72$ to 2.16$)$ & $1.18(1.10$ to 1.27$)$ & $1.62(1.45$ to 1.81$)$ & $1.43(1.23$ to 1.68$)$ \\
\hline \multicolumn{7}{|c|}{ Profession, ref=upper white-collar } \\
\hline Lower white-collar & $1.00(0.94$ to 1.07$)$ & 1.00 (0.93 to 1.07$)$ & 1.04 (0.91 to 1.18$)$ & $1.01(0.92$ to 1.11$)$ & 1.03 (0.92 to 1.15$)$ & $1.07(0.91$ to 1.26$)$ \\
\hline Blue-collar & 1.01 (0.96 to 1.06$)$ & 0.99 (0.94 to 1.04$)$ & 1.02 (0.93 to 1.12$)$ & $1.00(0.94$ to 1.07$)$ & $1.02(0.94$ to 1.11$)$ & 0.99 (0.88 to 1.12$)$ \\
\hline Others & 1.05 (0.96 to 1.14$)$ & $1.14(1.05$ to 1.25$)$ & $0.98(0.82$ to 1.16$)$ & $1.00(0.90$ to 1.12$)$ & $1.18(1.03$ to 1.34$)$ & $1.13(0.93$ to 1.36$)$ \\
\hline \multicolumn{7}{|c|}{ Income per household member, ref=quartile 4} \\
\hline Quartile 1 & 1.35 (1.27 to 1.44$)$ & 1.45 (1.35 to 1.56$)$ & 1.76 (1.54 to 2.02$)$ & 1.20 (1.11 to 1.30$)$ & $1.17(1.05$ to 1.30$)$ & $1.30(1.11$ to 1.53$)$ \\
\hline Quartile 2 & 1.25 (1.18 to 1.33$)$ & 1.34 (1.26 to 1.43$)$ & 1.44 (1.26 to 1.64$)$ & 1.13 (1.05 to 1.22$)$ & 1.15 (1.04 to 1.27$)$ & $1.23(1.06$ to 1.43$)$ \\
\hline Quartile 3 & 1.09 (1.03 to 1.16$)$ & $1.19(1.11$ to 1.27$)$ & 1.21 (1.05 to 1.38$)$ & 1.09 (1.01 to 1.17$)$ & 1.08 (0.98 to 1.19$)$ & 1.07 (0.92 to 1.24$)$ \\
\hline \multicolumn{7}{|c|}{ Diabetes medication, ref=yes } \\
\hline No & 1.12 (1.07 to 1.16$)$ & 1.09 (1.04 to 1.14$)$ & 1.09 (1.01 to 1.19$)$ & 1.09 (1.03 to 1.15$)$ & 1.03 (0.96 to 1.10$)$ & 1.07 (0.97 to 1.19$)$ \\
\hline \multicolumn{7}{|c|}{ Anticoagulants, ref=yes } \\
\hline No & 0.61 (0.59 to 0.64$)$ & 0.75 (0.73 to 0.78$)$ & 0.58 (0.54 to 0.63$)$ & 0.78 (0.73 to 0.83$)$ & 0.96 (0.89 to 1.03$)$ & 0.77 (0.68 to 0.86$)$ \\
\hline \multicolumn{7}{|c|}{ Antihypertensives, ref=yes } \\
\hline No & 0.87 (0.83 to 0.91$)$ & 0.93 (0.89 to 0.98$)$ & 0.85 (0.77 to 0.93$)$ & $0.93(0.86$ to 1.00$)$ & 1.02 (0.93 to 1.13$)$ & $0.98(0.85$ to 1.13$)$ \\
\hline \multicolumn{7}{|c|}{ Kidney disease, $r e f=n o$} \\
\hline Yes & 1.23 (1.13 to 1.34$)$ & 2.08 (1.96 to 2.21$)$ & 1.93 (1.72 to 2.16$)$ & $0.97(0.84$ to 1.11$)$ & $1.92(1.75$ to 2.11$)$ & $1.67(1.46$ to 1.92$)$ \\
\hline \multicolumn{7}{|c|}{ Cancer diagnosis $\ddagger$, ref=no } \\
\hline Yes & $1.02(0.94$ to 1.11$)$ & 1.87 (1.76 to 2.00$)$ & 1.12 (0.96 to 1.30$)$ & 1.09 (0.98 to 1.21$)$ & 1.38 (1.23 to 1.55$)$ & $1.08(0.90$ to 1.30$)$ \\
\hline Diabetes duration & & & & & & \\
\hline
\end{tabular}

Continued 
Table 3 Continued

\begin{tabular}{|c|c|c|c|c|c|c|}
\hline & \multicolumn{3}{|c|}{ Primary prevention, $\mathrm{n}=107587$} & \multicolumn{3}{|c|}{ Secondary prevention, $n=14327$} \\
\hline & CV events & All-cause mortality & CV mortality & CV events & All-cause mortality & CV mortality \\
\hline & HR (95\% Cl) & HR (95\% Cl) & HR (95\% Cl) & HR (95\% Cl) & HR (95\% Cl) & HR (95\% Cl) \\
\hline Per year & 1.01 (1.01 to 1.02$)$ & 1.01 (1.01 to 1.01$)$ & $1.02(1.01$ to 1.02$)$ & 1.01 (1.01 to 1.02$)$ & 1.01 (1.01 to 1.02$)$ & 1.02 (1.01 to 1.02$)$ \\
\hline \multicolumn{7}{|c|}{$\mathrm{HbA} 1 \mathrm{c}(\mathrm{mmol} / \mathrm{mol})$, ref=below 42} \\
\hline $42-52$ & 1.09 (1.03 to 1.15$)$ & 0.93 (0.88 to 0.99$)$ & 0.99 (0.88 to 1.12$)$ & $1.02(0.94$ to 1.10$)$ & 0.91 (0.82 to 1.01$)$ & $1.00(0.86$ to 1.17$)$ \\
\hline$>52$ & $1.29(1.21$ to 1.37$)$ & $1.10(1.04$ to 1.17$)$ & 1.27 (1.12 to 1.43$)$ & $1.21(1.11$ to 1.31$)$ & 1.10 (0.99 to 1.22$)$ & $1.25(1.07$ to 1.47$)$ \\
\hline \multicolumn{7}{|l|}{ eGFR, ref $=60$ or more } \\
\hline$<60 \mathrm{~m}$ & 1.19 (1.13 to 1.24$)$ & 1.22 (1.17 to 1.28$)$ & 1.31 (1.21 to 1.42$)$ & 1.16 (1.10 to 1.23$)$ & 1.33 (1.25 to 1.42$)$ & $1.42(1.29$ to 1.56$)$ \\
\hline \multicolumn{7}{|c|}{ Cholesterol levels, ref=LDL $<2.5, \mathrm{HDL}<1.0$ (men)/1.3 (women), triglycerides $<2.0$} \\
\hline $\mathrm{LDL} \geq 2.5$ & $1.22(1.17$ to 1.26$)$ & $0.93(0.90$ to 0.97$)$ & 1.05 (0.98 to 1.13$)$ & $1.08(1.03$ to 1.14$)$ & $1.02(0.96$ to 1.08$)$ & $1.14(1.04$ to 1.24$)$ \\
\hline $\begin{array}{l}\mathrm{HDL} \geq 1.0 \text { (men)/1.3 } \\
\text { (women) }\end{array}$ & 0.91 (0.88 to 0.95$)$ & $0.96(0.92$ to 1.00$)$ & 0.97 (0.90 to 1.05$)$ & 0.94 (0.89 to 0.99$)$ & $0.88(0.83$ to 0.94$)$ & $0.88(0.80$ to 0.97$)$ \\
\hline Triglycerides $\geq 2.0$ & 1.09 (1.05 to 1.13$)$ & 1.00 (0.96 to 1.04$)$ & 1.07 (0.99 to 1.15$)$ & 1.03 (0.97 to 1.08$)$ & 0.91 (0.85 to 0.97$)$ & $0.97(0.88$ to 1.07$)$ \\
\hline \multicolumn{7}{|l|}{ BMI, ref $=<25$} \\
\hline $25-29$ & 1.00 (0.95 to 1.05$)$ & $0.72(0.69$ to 0.75$)$ & 0.78 (0.71 to 0.85$)$ & 1.02 (0.96 to 1.09$)$ & $0.79(0.73$ to 0.85$)$ & 0.80 (0.71 to 0.89$)$ \\
\hline$\geq 30$ & $0.93(0.89$ to 0.98$)$ & $0.67(0.64$ to 0.70$)$ & 0.74 (0.68 to 0.82$)$ & $1.02(0.95$ to 1.10$)$ & $0.70(0.65$ to 0.76$)$ & 0.72 (0.64 to 0.82$)$ \\
\hline \multicolumn{7}{|c|}{ Blood pressure $(\mathrm{mm} \mathrm{Hg})$, ref=systolic $<130$, diastolic $<80$} \\
\hline Systolic $\geq 130$ & $1.14(1.10$ to 1.19$)$ & 0.84 (0.81 to 0.88$)$ & 0.93 (0.86 to 1.01$)$ & 0.96 (0.91 to 1.01$)$ & $0.81(0.76$ to 0.86$)$ & 0.80 (0.73 to 0.88$)$ \\
\hline Diastolic $\geq 80$ & 0.97 (0.94 to 1.01$)$ & 0.93 (0.90 to 0.97$)$ & $0.92(0.85$ to 0.98$)$ & 0.97 (0.92 to 1.02$)$ & 0.96 (0.90 to 1.02$)$ & 0.97 (0.88 to 1.06$)$ \\
\hline \multicolumn{7}{|c|}{ Microalbuminuria, ref=no } \\
\hline Yes & $1.16(1.11$ to 1.21$)$ & $1.26(1.21$ to 1.31$)$ & $1.32(1.22$ to 1.42$)$ & $1.08(1.02$ to 1.14$)$ & 1.19 (1.12 to 1.27$)$ & $1.19(1.08$ to 1.31$)$ \\
\hline \multicolumn{7}{|c|}{ Macroalbuminuria, ref=no } \\
\hline Yes & $1.28(1.21$ to 1.36$)$ & 1.40 (1.32 to 1.48$)$ & 1.56 (1.42 to 1.72$)$ & $1.16(1.07$ to 1.25$)$ & $1.38(1.27$ to 1.50$)$ & 1.38 (1.23 to 1.56$)$ \\
\hline \multicolumn{7}{|c|}{ Physical activity§, ref=daily } \\
\hline$<1$ time per week & 1.25 (1.20 to 1.31$)$ & 1.79 (1.71 to 1.88$)$ & 1.85 (1.69 to 2.03$)$ & 1.17 (1.10 to 1.25$)$ & 1.72 (1.59 to 1.87$)$ & 1.74 (1.55 to 1.96$)$ \\
\hline 1-2 times per week & 1.10 (1.05 to 1.15$)$ & 1.26 (1.20 to 1.33$)$ & $1.30(1.17$ to 1.44$)$ & 1.05 (0.98 to 1.12$)$ & 1.18 (1.07 to 1.29$)$ & 1.19 (1.04 to 1.38$)$ \\
\hline $3-5$ times per week & 0.99 (0.94 to 1.03$)$ & 1.04 (0.98 to 1.10$)$ & 0.98 (0.88 to 1.10$)$ & 1.00 (0.93 to 1.07$)$ & 1.05 (0.94 to 1.16$)$ & 1.04 (0.89 to 1.21$)$ \\
\hline \multicolumn{7}{|l|}{ Smokingף, ref=no } \\
\hline Yes & 1.16 (1.11 to 1.22$)$ & 1.38 (1.31 to 1.44$)$ & 1.38 (1.26 to 1.51$)$ & 1.07 (1.00 to 1.14$)$ & 1.17 (1.07 to 1.28$)$ & $1.23(1.08$ to 1.41$)$ \\
\hline
\end{tabular}

Adjusted HR (with $95 \% \mathrm{Cl}$ ) for any CV events, all-cause mortality and CV mortality for patient characteristics among patients with type 2 diabetes mellitus by prevention type.

*Includes Belgium, Denmark, Germany, Greece, Spain, France, Ireland, Italy, Luxembourg, The Netherlands, Austria, Portugal, Finland, Sweden and Great Britain. †lf age 65 years or older and unemployed.

$\ddagger$ Within 5 years prior to baseline.

$\S 30$ min walk or equivalent.

IAt least one cigarette or pipe per day or quit smoking within 3 months.

BMI, body mass index; CV, cardiovascular; HDL, high-density lipoprotein; HR, hazard ratio; HbA1c, hemoglobin A1c;IQR, interquartile range; LDL, low-density

lipoprotein; NA, not applicable; SD, standard deviation; TSEK, thousand Swedish Krona; eGFR, estimated glomerular filtration rate; ref, reference.

adherence to lipid-lowering prescription guidelines. To our knowledge, this is the first study that considers both refill and guideline adherence when analyzing the risk of $\mathrm{CV}$ events and mortality among patients with T2DM.

We found that patients with low adherence to lipid-lowering medications had a higher risk of CV events and mortality in both prevention groups, independent of the center's guideline adherence level. Furthermore, low-adherent primary prevention patients who were being treated at low-adherent centers faced a higher risk of CV events and CV mortality. Otherwise, guideline adherence had little or no effect on the risk of CV events or mortality in either prevention group. This suggests that patient adherence was the major factor with respect to lipid-lowering prevention of $\mathrm{CV}$ events and mortality among patients with T2DM.

Our results concur with previous studies that have shown that high refill adherence to lipid-lowering medications are associated with a lower risk of $\mathrm{CV}$ events and mortality than low refill adherence. ${ }^{581213}$ Furthermore, we have previously shown that among multiple levels of refill adherence, the risk of CV events gradually increases with lower refill adherence. ${ }^{6}$ For those reasons, the healthcare center should be observant in order to detect early signs ofrefill non-adherence.

We found several patient characteristics associated with a higher risk of CV events and mortality in both prevention groups: male gender, increasing age, unemployment, 
Primary prevention

Cardiovascular events
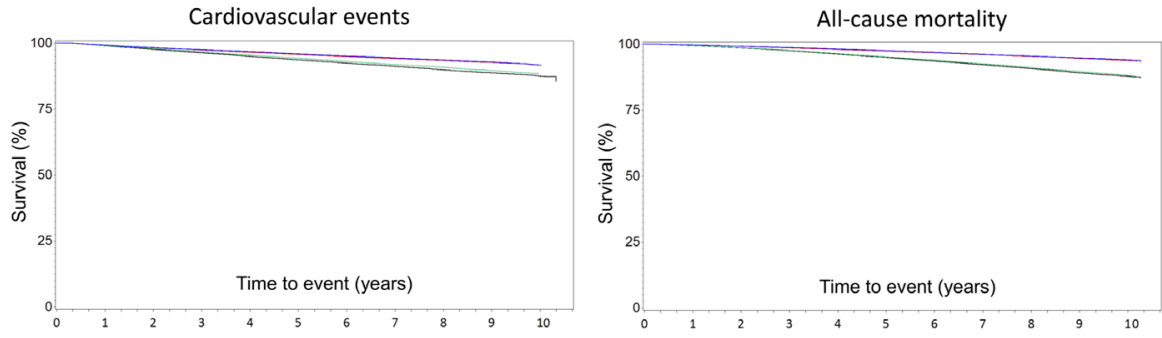

Secondary prevention
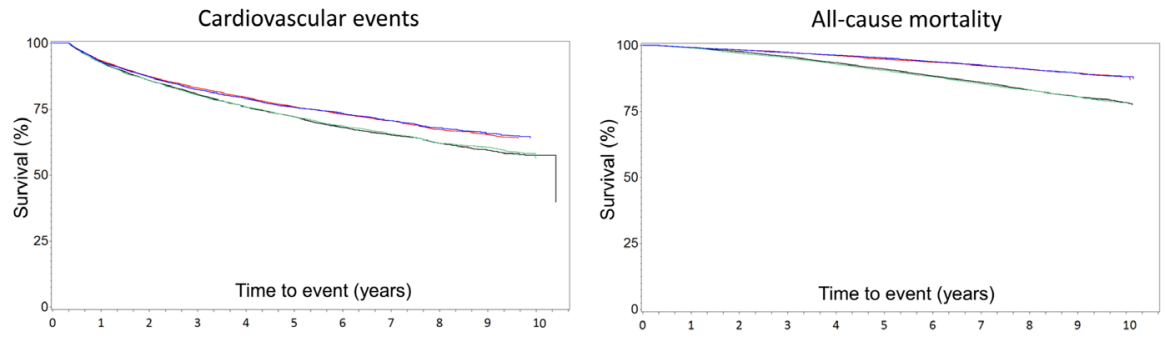

Cardiovascular mortality
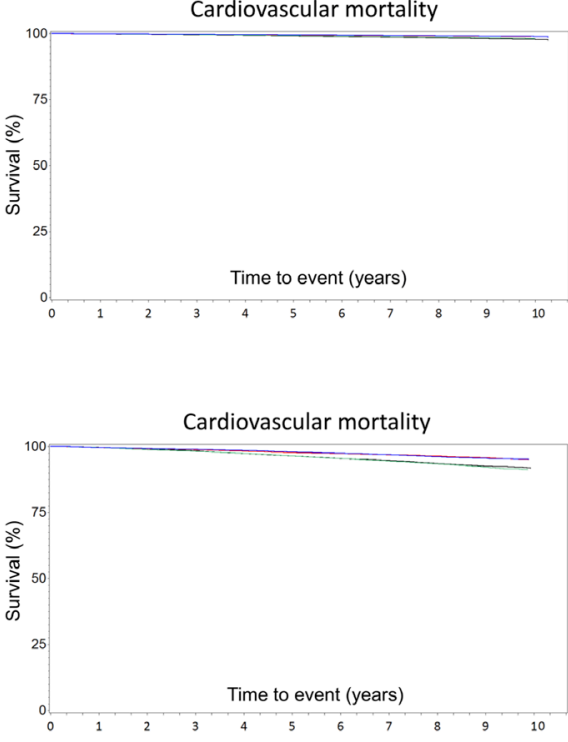

- LOW refill adherence LOW guideline adherence $\quad$ HIGH refill adherence LOW guideline adherence $\quad$ LOW refill adherence HIGH guideline adherence $\quad$ HIGH refill adherence HIGH guideline adherence

Figure 2 Adjusted Kaplan-Meier survival curves for cardiovascular (CV) events, all-cause mortality and CV mortality for patients with type 2 diabetes mellitus by prevention type.

lower income, higher HbAlc, lower kidney function, and so on. These characteristics have previously been shown to be associated with a higher risk of CVD and mortality in the general population ${ }^{36}$ and among patients with T2DM. ${ }^{6}$ Furthermore, we found patients without anticoagulants and antihypertensives, as well as those born outside of Sweden, to have lower risk of CV events and mortality. This differs from previous studies that have shown non-adherence to cardioprotective medications as associated with higher risk of CVD and mortality. ${ }^{37} 38$ However, a closer look reveals that patients without other cardioprotective medications and those born in Africa, the Americas, Oceania or Asia were younger, had shorter diabetes duration and less comorbidities, as well as shorter follow-up time for those born outside of Sweden. This could explain the lower risk of CV events and mortality in the present study. Our findings suggest that individual risk of CVD should be considered when choosing a diabetes treatment regimen.

Although adherence to lipid-lowering prescription guidelines had little or no impact on the risk of CV events and mortality, we have not considered nurses, dietitians, physical therapists, pharmacists, and so on, with whom patients with diabetes come in contact. Caregiver attitudes and beliefs concerning diabetes management have been shown to influence self-management behavior among patients with T2DM. ${ }^{39}{ }^{40}$ Furthermore, patients with T2DM who had access to a diabetes team, group training programs and diabetes nurses had better control of their HbAlc. ${ }^{40}$

Low guideline adherence is not synonymous with low quality of care. In the present study, guideline adherence represented the prevalence of prescribed lipid-lowering medication among patients with T2DM with LDL cholesterol above the recommended target values entered in the NDR. We did not know why medications were not prescribed. Previous studies have shown that prescription of lipid-lowering medications for patients with T2DM is usually based on the individual risk of developing CVD rather than LDL cholesterol alone. ${ }^{14}{ }^{15}{ }^{17-19}$ Current treatment guidelines take a similar approach. ${ }^{13941}$

\section{Strengths and limitations}

The greatest strengths of this study are its national coverage and the linkage of individual data among national registers. By Swedish law, reporting to the National Patient Register and Cause of Death Register is mandatory; under-reporting is estimated at less than $1 \%$. Thus, the results of this study tally well with clinical practice. Furthermore, the SPDR allowed an assessment of patient adherence using data about filled prescriptions instead of data about prescriptions that may or may not have been filled. However, we cannot be sure that patients actually took their medication, which constitutes the major limitation of our study.

As a result, we started following each patient when the first supply had ceased, that is, the first day of possible non-adherence. That eliminated the uncertainty concerning adherence that accompanies the first filled prescription, as it will always appear to be complete regardless of subsequent behavior.

Another limitation of our study is missing data, especially from the NDR. Not all patient characteristics are measured at every appointment, and most patients with T2DM have only an annual check-up. Nonetheless, the NDR includes data from both primary and specialized 
care clinics, permitting a population sample representative of patients in clinical practice. Furthermore, a sensitivity analysis between imputed data and complete cases showed similar results. Thus, the impact of missing data on our final conclusions appears to be minimal.

Since the National Patient Register does not cover diagnoses in primary care, only kidney disease diagnosed in inpatient or outpatient care was included. However, several other measurements of kidney function were accounted for (eGFR, microalbuminuria, macroalbuminuria, and so on). Thus, we believe that the study fully covered kidney disease as a risk factor.

Adverse drug reactions and contraindications would be legitimate reasons to abstain from prescribing lipid-lowering medications for patients with T2DM. However, no such information was considered. Thus, we may have included patients who were unsuitable for lipid-lowering medications. Furthermore, excluding patients who experienced CV events or died during their first filled supply or interval ( $\mathrm{n}=15425)$ may have introduced a selections bias that excluded the most fragile patients.

There was a statistically significant association between refill and guideline adherence. However, the association was too small to be clinically relevant. Thus, guideline adherence was assumed to have little or no impact on refill adherence and the variables were regarded as independent.

A comparison between the SPDR and the NDR regarding the healthcare center at baseline showed high conformity. The classification of type of care in the NDR was dichotomized as primary or specialized care, while the type of care in the SPDR was broken down into 19 categories. This may explain the lower agreement with respect to type of care.

\section{CONCLUSIONS}

Patient refill adherence to lipid-lowering medications had a greater impact on the risk of CV events and mortality than healthcare center adherence to lipid-lowering guidelines or prevention type. These results demonstrate the value of individualized care among patients with T2DM, for example, through educational programs and risk factor counseling.

\section{Author affiliations}

${ }^{1}$ Department of Public Health and Community Medicine, Institute of Medicine, Sahlgrenska academy, University of Gothenburg, Goteborg, Sweden ${ }^{2}$ Department of Molecular and Clinical Medicine, Institute of Medicine, Sahlgrenska Academy, University of Gothenburg, Gothenburg, Sweden

${ }^{3}$ National Diabetes Register, Centre of Registers, Gothenburg, Sweden

${ }^{4}$ Global Medical Affairs, Medical Evidence and Observational Research, AstraZeneca AB, Mölndal, Sweden

Acknowledgements We wish to acknowledge Ken Schubert, Twenty-first Century Translation, for the language editing of the manuscript.

Contributors SAK, BE, SF, MM, A-MS and KAS contributed to the study design and the implementation of the research; to the analysis and the implementation of the results; and the writing of the manuscript. SAK and SF performed the statistical analyses. SAK and KAS drafted the manuscript. KAS is the guarantor of the study.
Funding This study was funded by a grant from the Swedish Research Council for Health, Working Life and Welfare (\#2013-0521).

Disclaimer The funder had no role in the study design or decision to submit the manuscript for publication.

Competing interests KAS is employed by AstraZeneca. However, the views expressed in this article are her own and not those of AstraZeneca's. All other authors declare that they have no competing interests associated with their contribution to the manuscript.

Patient consent for publication Not required.

Provenance and peer review Not commissioned; externally peer reviewed.

Data sharing statement No additional data are available.

Open access This is an open access article distributed in accordance with the Creative Commons Attribution 4.0 Unported (CC BY 4.0) license, which permits others to copy, redistribute, remix, transform and build upon this work for any purpose, provided the original work is properly cited, a link to the licence is given, and indication of whether changes were made. See: https://creativecommons.org/ licenses/by/4.0/.

\section{REFERENCES}

1. American Diabetes Association. 9. Cardiovascular disease and risk management. Diabetes Care 2017;40(Supplement 1):S75-S87.

2. American Diabetes Association. 6. Glycemic Targets: Standards of Medical Care in Diabetes-2018. Diabetes Care 2018;41(Supplement 1):S55-S64.

3. International Diabetes Federation. Global guideline for type 2 diabetes. Brussels: International Diabetes Federation, 2012.

4. Rawshani A, Rawshani A, Franzén S, et al. Mortality and cardiovascular disease in type 1 and type 2 diabetes. N Engl $\mathrm{J}$ Med 2017;376:1407-18.

5. Ruokoniemi P, Korhonen MJ, Helin-Salmivaara A, et al. Statin adherence and the risk of major coronary events in patients with diabetes: a nested case-control study. Br J Clin Pharmacol 2011;71:766-76.

6. Karlsson SA, Hero C, Svensson A-M, et al. Association between refill adherence to lipid-lowering medications and the risk of cardiovascular disease and mortality in Swedish patients with type 2 diabetes mellitus: a nationwide cohort study. BMJ Open 2018;8:e020309.

7. de Vries FM, Denig P, Pouwels KB, et al. Primary prevention of major cardiovascular and cerebrovascular events with statins in diabetic patients. Drugs 2012;72:2365-73.

8. Deera MA B V, Burns LC, et al. Impact of statin adherence on cardiovascular disease and mortality outcomes: a systematic review. Br J Clin Pharmacol 2014;78:684-98.

9. National Board of Health and Welfare. Nationella riktlinjer för diabetesvården 2018 - Stöd för styrning och ledning. Socialstyrelsen, 2018.

10. International Diabetes Federation. IDF clinical practice recommendations for managing type 2 diabetes in primary care. Brussels: International Diabetes Federation, 2017.

11. World Health Organization. Adherence to long-term therapies, evidence for action. Geneva: World Health Organization, 2003.

12. Korhonen MJ, Ruokoniemi P, llomäki J, et al. Adherence to statin therapy and the incidence of ischemic stroke in patients with diabetes. Pharmacoepidemiol Drug Saf 2016;25:161-9.

13. $\mathrm{PM} \mathrm{H}$, Magid DJ, Masoudi FA, et al. Adherence to cardioprotective medications and mortality among patients with diabetes and ischemic heart disease. BMC Cardiovasc Disord 2006;6.

14. Wu J, Zhu S, Yao GL, et al. Patient factors influencing the prescribing of lipid lowering drugs for primary prevention of cardiovascular disease in UK general practice: a national retrospective cohort study. PLoS ONE 2013;8:e67611.

15. Jones NRV, Fischbacher CM, Guthrie B, et al. Factors associated with statin treatment for the primary prevention of cardiovascular disease in people within 2 years following diagnosis of diabetes in Scotland, 2006-2008. Diabet. Med. 2014;31:640-6.

16. Eriksson M, Zethelius B, Eeg-Olofsson $\mathrm{K}$, et al. Blood lipids in 75,048 type 2 diabetic patients: a population-based survey from the Swedish national diabetes Register. European Journal of Cardiovascular Prevention \& Rehabilitation 2011;18:97-105.

17. Berthold HK, Gouni-Berthold I, Böhm M, et al. Patterns and predictors of statin prescription in patients with type 2 diabetes. Cardiovasc Diabetol 2009;8.

18. Thomas MC, Nestel PJ. Management of dyslipidaemia in patients with type 2 diabetes in Australian primary care. Med J Aust 2007;186:128-30. 
19. Pokharel Y, Akeroyd JM, Ramsey DJ, et al. Statin use and its Facility-Level variation in patients with diabetes: insight from the Veterans Affairs national database. Clin Cardiol 2016;39:185-91.

20. Wettermark B, Hammar N, MichaelFored C, et al. The new Swedish prescribed drug Register-Opportunities for pharmacoepidemiological research and experience from the first six months. Pharmacoepidem. Drug Safe. 2007;16:726-35.

21. Eliasson B, Gudbjörnsdottir S. Diabetes care - improvement through measurement. Diabetes Research and Clinical Practice 2014;106:S2 91-S294.

22. National Board of Health and Welfare. The National patient register. Available: http://www.socialstyrelsen.se/register/ halsodataregister/patientregistret/inenglish [Accessed $01 \mathrm{Apr}$ 2015].

23. National Board of Health and Welfare. Cause of death register. Available: http://www.socialstyrelsen.se/statistics/ statisticaldatabase/help/causeofdeath [Accessed 01 Apr 2015].

24. The National Board of Health and Welfare. Swedish Cancer registry. Available: http://www.socialstyrelsen.se/register/halsodataregister/ cancerregistret/inenglish [Accessed 16 May 2017].

25. Statistics Sweden. Longitudinal integration database for health insurance and labour market studies (LISA by Swedish acronym). Available: http://www.scb.se/lisa-en [Accessed 01 Apr 2015].

26. World Health Organization. Definition and diagnosis of diabetes mellitus and intermediate hyperglycemia. Geneva: World Health Organization, 2006.

27. Scaldaferri F, Pizzoferrato M, Ponziani FR, et al. Use and indications of cholestyramine and bile acid sequestrants. Intern Emerg Med 2013;8:205-10.

28. Karlsson SA, Hero C, Eliasson B, et al. Refill adherence and persistence to lipid-lowering medicines in patients with type 2 diabetes: a nation-wide register-based study. Pharmacoepidemiol Drug Saf 2017;26:1220-32.

29. Chowdhury R, Khan H, Heydon E, et al. Adherence to cardiovascular therapy: a meta-analysis of prevalence and clinical consequences. Eur Heart J 2013;34:2940-8.

30. Cramer JA, Benedict Á., Muszbek N, et al. The significance of compliance and persistence in the treatment of diabetes, hypertension and dyslipidaemia: a review. Int J Clin Pract 2008;62:76-87.

31. National Board of Health and Welfare. Nationella riktlinjer för diabetesvården 2010 - Stöd för styrning och ledning. Västerås: Socialstyrelsen, 2010.

32. World Health Organization. Body mass index - BMI. Available: http:// www.euro.who.int/en/health-topics/disease-prevention/nutrition/ahealthy-lifestyle/body-mass-index-bmi [Accessed 03 Mar 2016].

33. National Kidney Foundation. Glomerular filtration rate (GFR). Available: https:// www.kidney.org/atoz/content/gfr [Accessed 21 May 2016].

34. R Core Team. R: a language and environment for statistical computing Vienna, Austria, 2013. Available: http://www.R-project. org/

35. Buuren Svan, Groothuis-Oudshoorn K. mice : Multivariate Imputation by Chained Equations in R. Journal of Statistical Software 2011;45:1-67.

36. Havranek EP, Mujahid MS, Barr DA, et al. Social determinants of risk and outcomes for cardiovascular disease: a scientific statement from the American Heart Association. Circulation 2015;132:873-98.

37. PM H, Magid DJ, Shetterly SM, et al. Medication nonadherence is associated with a broad range of adverse outcomes in patients with coronary artery disease. Am Heart J 2008;155:772-9.

38. PM H, Rumsfeld JS, Masoudi FA, et al. Effect of medication nonadherence on hospitalization and mortality among patients with diabetes mellitus. Arch Intern Med 2006;166:1836-41.

39. Nam S, Chesla C, Stotts NA, et al. Barriers to diabetes management: patient and provider factors. Diabetes Research and Clinical Practice 2011;93:1-9.

40. Husdal R, Rosenblad A, Leksell J, et al. Resources and organisation in primary health care are associated with $\mathrm{HbA} 1 \mathrm{c}$ level: a nationwide study of 230958 people with type 2 diabetes mellitus. Prim Care Diabetes 2018;12:23-33

41. Stone NJ, Robinson JG, Lichtenstein AH, et al. 2013 ACC/ AHA guideline on the treatment of blood cholesterol to reduce atherosclerotic cardiovascular risk in adults: a report of the American College of Cardiology/American Heart Association Task Force on practice guidelines. Circulation 2014;129(25 Suppl 2):S1-45. 